\title{
Association between perceived health risks of e-cigarettes and actual e-cigarette use, based on cigarette smoking status and sexual and gender minority status among U.S. adults
}

\author{
David Adzrago ${ }^{1}$ (D) Yue Shi ${ }^{1} \cdot$ Kayo Fujimoto ${ }^{1}$
}

Received: 10 June 2021 / Accepted: 7 November 2021

This is a U.S. government work and not under copyright protection in the U.S.; foreign copyright protection may apply 2021

\begin{abstract}
Background There is a lack of consensus in the literature about the association between the perceived health risks of e-cigarettes and their actual use, an association that may be based on cigarette smoking status or sexual and gender minority status. This study examined the moderating effect of these variables as well as differences in e-cigarette use between and within perceived harmfulness of e-cigarettes and cigarette smoking among U.S. adults.

Methods The data were drawn from the 2020 and 2019 Health Information National Trends Survey (HINTS 5), Cycles 4 and 3 , on adults $(N=9303)$. We estimated weighted multivariable logistic regression models and conducted marginal analyses based on perceived harmfulness of e-cigarettes.

Results Of the study population, $30.75 \%$ currently used e-cigarettes daily or some days. No significant difference in e-cigarette use was found between sexual minorities and heterosexuals, but there was a significant interaction between sexual identity and perceived harmfulness of e-cigarettes. Compared to non-smokers, current daily or some days cigarette smokers were more likely to use e-cigarettes ( $\mathrm{AOR}=3.48,95 \% \mathrm{CI}=1.25,9.72$ ). Those who perceived e-cigarettes to be just as harmful as actual cigarettes $(\mathrm{AOR}=0.13,95 \% \mathrm{CI}=0.06,0.27)$ or more harmful $(\mathrm{AOR}=0.03,95 \% \mathrm{CI}=0.01,0.10)$, or were uncertain $(\mathrm{AOR}=0.18,95 \%$ $\mathrm{CI}=0.08,0.45$ ), were less likely to use e-cigarettes compared to those who perceived e-cigarettes to be less harmful. Former and current cigarette smokers who perceived e-cigarettes as less harmful had a higher probability of using e-cigarettes daily or some days than did non-cigarette smokers. For the current and former cigarette smoking groups, those who perceived e-cigarettes as less harmful had the highest probability of current e-cigarette use than did those who were uncertain or perceived e-cigarettes as just as harmful, whereas the probability was not significant for those who perceived e-cigarettes as more harmful.

Conclusion Current daily or some days use of e-cigarettes is associated with current daily or some days cigarette smoking and lowered perceived harmfulness of e-cigarettes among the U.S. adult population. Tailored health promotion and intervention efforts may reduce the potential perceived health and behavioral/lifestyle risks related to the use of tobacco products, especially the use of e-cigarettes daily among former and current cigarette smokers as well as those who perceive e-cigarettes as less harmful.
\end{abstract}

Keywords E-cigarettes $\cdot$ Sexual and gender minorities $\cdot$ Cigarette smoking $\cdot$ Perceived health risks

\section{Introduction}

E-cigarette (also known as electronic cigarettes or electronic nicotine delivery systems [ENDS]) use involves the inhaling of vapors of nicotine, flavor, and other chemicals that

David Adzrago

David.Adzrago@uth.tmc.edu

1 Center for Health Promotion and Prevention Research, The University of Texas Health Science Center at Houston, 7000 Fannin, Suite 2502A, Houston, TX 77030, USA are delivered through battery-operated devices that deliver the vapors (Grana et al. 2014; Pericot-Valverde et al. 2017; Tan and Bigman 2014). These battery-operated devices include disposable, rechargeable, pen-style medium-sized rechargeable, and tank-style large-sized rechargeable formats (Centers for Disease Control and Prevention [CDC], 2020; Grana et al. 2014). Ever use of e-cigarettes has increased from 1.8\% in 2010 to $14.9 \%$ in 2018 (McMillen et al. 2014; Pericot-Valverde et al. 2017; Villarroel et al. 2020), whereas current use increased from $0.3 \%$ in 2010 to $4.5 \%$ in 2019 (Cornelius et al. 2020; McMillen et al. 2014). Nicotine is a highly addictive and harmful chemical in tobacco products, 
including e-cigarettes, cigars, cigarettes, and other tobacco products (CDC 2020; Singh et al. 2020; U.S. Department of Health and Human Services 2016). Because approximately $99 \%$ of e-cigarettes contain nicotine (CDC 2020; Singh et al. 2020; US Department of Health and Human Services 2016), e-cigarette use has negative health risks for the pulmonary system and cardiovascular systems as well as a carcinogenic effect (Pisinger and Døssing 2014). According to CDC (2020), e-cigarette use results in poisoning and lung disease and harms brain development in adolescents and young adults. The safety, efficacy for harm reduction and cessation, and overall impact of e-cigarette use on public health, however, have not been established (Douglass et al. 2020; Grana et al. 2014). Thus, there is a need to improve our understanding of the interaction of the perceived health risks of e-cigarette use, actual e-cigarette use, and being a member of an at-risk population, to effectively design and implement evidence-based tobacco prevention, reduction, and cessation-promotion interventions.

There is a lack of consensus in the literature about the associations between the perceived health risks of e-cigarettes and their actual use among U.S. adults (Cooper et al. 2016; Czoli et al. 2017; Manzione et al. 2020; Piñeiro et al. 2016; Popova et al. 2017; Wackowski and Delnevo 2016). Individuals who perceived e-cigarettes as less harmful than actual cigarettes are more likely to use e-cigarettes (Amrock et al. 2016; Huang et al. 2019; Manzione et al. 2020). E-cigarette users, perhaps being influenced by external forces such as multinational tobacco companies and marketers, including some celebrities, often perceive e-cigarettes as less harmful and as substitutes for quitting or reducing tobacco intake via cigarette smoking and other tobacco products (Cobb et al. 2015; Grana et al. 2014; Luo et al. 2014; Noel et al. 2011; Paek et al. 2014; U.S. National Cancer Institute 2017). Other research has found that e-cigarette users often correctly perceive the health harmfulness of e-cigarettes as compared to non-users (Czoli et al. 2017).

The association between risk perceptions of the harmfulness of the use of e-cigarettes and their actual use may depend on cigarette smoking status. Some studies reported that adult cigarette smokers perceived e-cigarettes as less harmful than cigarette smoking and, therefore, felt that a switch to e-cigarette use would have lower harms (National Academies of Sciences, Engineering, and Medicine 2018; Popova et al. 2018; U.S. Department of Health and Human Services 2014). There are, however, mixed findings with regard to the moderation of cigarette smoking status on the association between perceptions of harmfulness of e-cigarettes and their actual use. For example, those who perceive e-cigarettes as less harmful are more likely to smoke cigarettes and use e-cigarettes (Ganz et al. 2018; Popova et al. 2018), suggesting that, if cigarette smokers perceive e-cigarettes as less harmful, they are more likely to use e-cigarettes as well. Another study (Perski et al.
2020), however, found that cigarette smokers were less likely to use e-cigarettes, even if they perceive e-cigarettes as less harmful.

In addition to the cigarette smoking status, the association between risk perceptions of e-cigarettes and their actual use may depend on sexual and gender minority (SGM) (LGBTQ; lesbian, gay, bisexual, transgender, and queer) status. It has been reported that SGM adults are more likely to use any tobacco products, including e-cigarettes, than non-SGM adults (heterosexuals) (Wheldon and Wiseman 2019). In view of the higher prevalence of e-cigarette use among the SGM adults compared to their non-SGM counterparts (American Psychological Association 2015; Emory et al. 2016), SGM adults are at an increased risk of e-cigarette-related negative health outcomes than non-SGM adults (Buchting et al. 2017; Delahanty et al. 2019; Jamal et al. 2018; Ortiz et al. 2017). SGM status may be associated with lower e-cigarette harm perceptions due to the higher prevalence of this perception among SGM adults (44.6\%) compared to their non-SGM counterparts (37.3\%) (Ganz et al. 2018; Nayak et al. 2017). This association, however, is not supported by the findings of no difference in the level of perceived harmfulness of e-cigarettes between SGM adults and non-SGM adults (Nayak et al. 2017). Likewise, another study did not find any differences between SGM and non-SGM adults in regard to the level of perceived harmfulness of e-cigarettes associated with recent e-cigarette use (Ganz et al. 2018). These studies indicate that, although there is a high prevalence of e-cigarette use among SGM adults, the perceived harmfulness of e-cigarettes may not explain their actual use.

To address this gap in the existing literature related to the perception of the risk of e-cigarette use, actual e-cigarette use, cigarette smoking, and SGM status, our study examines, through the use of a nationally representative sample of the U.S. adult population, whether the perceived harmfulness of e-cigarettes is associated with their actual use and, if so, whether the association is based on the status of cigarette smoking or SGM.

\section{Methods}

\section{Study design}

Data were drawn from the 2020 and 2019 Health Information National Trends Survey (HINTS) de-identified publicuse data. The HINTS is a nationally representative annual cross-sectional survey that has been sponsored by the National Cancer Institute (NCI) since 2003. It is conducted among adults aged 18 years or older in the United States. Multistage random sampling is used to recruit for the HINTS a sample of the U.S. civilian, noninstitutionalized adult population. Details of the methods and survey questions 
can be found in Finney Rutten et al. (2020) and the Health Information National Trends Survey 5 (HINTS 5) Cycle 4 report (Westat 2020). HINTS assesses U.S. adults' access to and use of health-related information and health-related behaviors, perceptions, and knowledge. We used the most recent HINTS data, HINTS 5 Cycles 4 and 3, which were conducted between February through June 2020 and January through April 2019, respectively. The HINTS 5 Cycles 4 and 3 data consist of a total sample of 9303 adults. Because we used the HINTS de-identified publicly available data, the authors' Institutional Review Board determined that this study is not a research with human subjects and therefore qualified for an exemption (RE: HSC-SPH-21-0784).

\section{Measures}

\section{Response variable}

The response variable is e-cigarette use, which was measured by asking the participants to indicate whether they now use an e-cigarette every day, some days, or not at all (Yes/ No). This variable was dichotomized to indicate e-cigarette use status as "yes" (current e-cigarette use) and "no" (not currently using e-cigarettes).

\section{Explanatory variable}

Perceived harmfulness of e-cigarettes is the explanatory variable. The participants were asked to indicate whether e-cigarettes, compared to cigarettes, are much less harmful, less harmful, just as harmful, more harmful, much more harmful, and do not know. We recoded these categories as less harmful (much less harmful + less harmful), just as harmful, more harmful (more harmful + much more harmful), and uncertain (i.e., do not know).

\section{Covariates}

Based on the literature (American Psychological Association 2015; Emory et al. 2016; Ganz et al. 2018; Nayak et al. 2017), self-reported sociodemographic characteristics and cigarette smoking status were adjusted for in the analyses. These variables include age (18-25, 26-34, 35-49, 50-64, and 65+), sex (male and female), race/ethnicity (non-Hispanic White, non-Hispanic Black/African American, Hispanic, and nonHispanic others), sexual orientation (heterosexual/straight and sexual minorities [homosexual, lesbian, gay, or bisexual]), level of education completed (coded as ordered categorical variable [less than high school, high school graduate, some college, and college graduate or higher]), total family income (coded as an ordered categorical variable $[<\$ 20,000, \$ 20,000$ to $<\$ 35,000, \$ 35,000$ to $<\$ 50,000, \$ 50,000$ to $<\$ 75,000$, and $\geq \$ 75,000]$ ), census region (Northeast, Midwest, West, and South), general health status (coded as a binary variable [excellent/very good/good and fair/poor]), and cigarette smoking status (non-smoker, former smoker, and current daily or some days smoker). Cigarette smoking status was derived from two survey questions: (1) Have you smoked at least 100 cigarettes in your entire life? (yes/no) and (2) How often do you now smoke cigarettes? (every day, some days, or not at all). Those who had never smoked at least 100 cigarettes in their entire life were categorized as never-smokers; lifetime users who do not smoke cigarettes now were categorized as former smokers; and lifetime cigarette smokers who currently smoke cigarettes every day or some days were categorized as daily or some days cigarette smokers.

\section{Statistical analyses}

First, we estimated the prevalence of e-cigarette use by the participants' sociodemographic characteristics and perceived harmfulness of e-cigarettes. Second, we performed multivariable logistic regression analysis to assess the association between the perceived harmfulness of e-cigarettes and e-cigarette use, adjusting for the sociodemographic characteristics. Third, we tested the interaction effect of perceived harmfulness of e-cigarettes and cigarette smoking status to assess the potential modification of cigarette smoking on the association. Likewise, we also tested the interaction of SGM status and perceived harmfulness of e-cigarettes. We further performed marginal analyses using margins command in STATA, to examine differences in e-cigarette use between and within cigarette smoking status and the perceived harmfulness of e-cigarettes and sexual identity and the perceived harmfulness of e-cigarettes. All of the statistical analyses were conducted at an alpha level of 0.05 (two-tailed), 95\% confidence intervals ( $95 \% \mathrm{CIs}$ ), and adjusted odds ratios (AORs), using STATA version 16.1 (StataCorp 2019). The analyses also were weighted using the HINTS survey weight to achieve nationally representative estimates (Finney Rutten et al. 2020; Westat 2020).

\section{Results}

\section{Descriptive statistics}

The descriptive statistics of the study sample (Weighted $N=95,069,931$ ) are presented in Table 1. Overall, 26.16\% of the participants were in the age range of 18-25 years and $26.44 \%$ were in the age range of $35-49$ years; $56.45 \%$ were males; $71.03 \%$ were non-Hispanic Whites; $89.54 \%$ were heterosexuals; $47.39 \%$ had some college education; $35.68 \%$ had a family income of $\$ 75,000$ or more; and $40.50 \%$ resided in the South. Of the participants, $16.74 \%$ had fair or poor general health, $37.08 \%$ were current daily or some days cigarette 
smokers, $35.42 \%$ perceived e-cigarettes to be less harmful, and $30.75 \%$ currently used e-cigarettes daily or some days. Among the e-cigarette users, $36.89 \%$ were in the age range of $18-25$ years; $56.07 \%$ were males; $75.59 \%$ were non-Hispanic Whites; $45.36 \%$ had some college education; $34.97 \%$ had a family income of $\$ 75,000$ or more; and $36.77 \%$ resided in the South. Of the current e-cigarette users, $9.75 \%$ were sexual minorities; $10.26 \%$ had fair or poor general health; $37.95 \%$ were current cigarette smokers; and $63.36 \%$ perceived e-cigarettes to be less harmful than cigarettes.

\section{Multivariable logistic regression results}

Table 2 presents the weighted multivariable logistic regression analysis results. Compared to individuals aged 18-25 years, individuals in the age range of 26-34 years $(\mathrm{AOR}=0.32,95 \% \mathrm{CI}=0.11,0.88)$, $50-64$ years $(\mathrm{AOR}=0.23,95 \% \mathrm{CI}=0.07,0.77)$, and $65+$ years $(\mathrm{AOR}=0.17,95 \% \mathrm{CI}=0.03,0.80)$ were less likely to engage in current e-cigarette use. Individuals who selfreported their general health as fair or poor also were less likely to use e-cigarettes $(\mathrm{AOR}=0.32,95 \% \mathrm{CI}=0.15,0.69)$ relative to those who reported their general health as excellent/very good/good. Compared to non-cigarette smokers, current cigarette smokers had higher odds of using e-cigarettes $(\mathrm{AOR}=3.48,95 \% \mathrm{CI}=1.25,9.72)$. Those who perceived e-cigarettes to be just as harmful ( $\mathrm{AOR}=0.13,95 \%$ $\mathrm{CI}=0.06,0.27)$, more harmful $(\mathrm{AOR}=0.03,95 \% \mathrm{CI}=0.01$, $0.10)$, or uncertain $(\mathrm{AOR}=0.18,95 \% \mathrm{CI}=0.08,0.45)$ were more likely to use e-cigarettes compared to those who perceived e-cigarettes to be less harmful than cigarette smoking. The interaction between cigarette smoking status and perceived harmfulness of e-cigarettes was statistically significant $(p<0.001)$, indicating that sexual identity modified the association between perceived harmfulness of e-cigarettes and actual e-cigarette use. We also found a significant interaction between sexual identity and perceived harmfulness of e-cigarettes $(p<0.001)$. Therefore, we proceeded to conduct marginal analyses. The analyses are presented below.

\section{Between and within-group analysis}

We examined differences in the association between e-cigarette use and perceived harmfulness of e-cigarettes by cigarette smoking status. Figure 1 presents the differences in e-cigarette use between and within cigarette smoking status and perceived harmfulness of e-cigarettes. Individuals who were former cigarette users and perceived e-cigarettes as less harmful than cigarettes (margin $=0.72,95 \% \mathrm{CI}=0.59,0.85$ ) had the highest probability of currently using e-cigarettes compared to current cigarette users (margin $=0.64,95 \%$ $\mathrm{CI}=0.45,0.84)$ and non-cigarette smokers (margin $=0.39$, $95 \% \mathrm{CI}=0.23,0.55$ ), who also perceived e-cigarettes as less harmful than smoking cigarettes. For current cigarette smokers, those who perceived e-cigarettes as less harmful than cigarettes had a higher probability of current e-cigarette use (margin $=0.64,95 \% \mathrm{CI}=0.45,0.84)$ than those who were uncertain $($ margin $=0.37,95 \% \mathrm{CI}=0.17,0.57$ ) or perceived e-cigarettes as just as harmful (margin $=0.31,95 \% \mathrm{CI}=0.16$, 0.47 ); the probability was not significant, however, for those who perceived e-cigarettes as more harmful (margin $=0.16$, $95 \% \mathrm{CI}=-0.05,0.28$ ). For former smokers, those who perceived e-cigarettes as less harmful had a higher probability of current e-cigarette use (margin $=0.72,95 \% \mathrm{CI}=0.59$, 0.85 ) than did those who were uncertain (margin $=0.22,95 \%$ $\mathrm{CI}=0.01,0.42$ ) or perceived e-cigarettes as just as harmful (margin $=0.16,95 \% \mathrm{CI}=0.01,0.30)$, while the probability was not significant for those who perceived e-cigarettes as more harmful (margin $=0.04,95 \% \mathrm{CI}=-0.01,0.09)$. For the non-cigarette smokers, the probability of current e-cigarette use was higher for those who perceived e-cigarettes as less harmful (margin $=0.39,95 \% \mathrm{CI}=0.23,0.55)$ than those who perceived it as just as harmful (margin $=0.15$, $95 \% \mathrm{CI}=0.02,0.29$ ), whereas the probabilities were not significant for those who perceived e-cigarettes as more harmful (margin $=0.05,95 \% \mathrm{CI}=-0.07,0.17$ ) or were uncertain $(\operatorname{margin}=0.23,95 \% \mathrm{CI}=-0.15,0.61)$.

Differences in the association between e-cigarette use and perceived harmfulness of e-cigarettes by sexual identity are presented in Fig. 2. The probability of current e-cigarette use was higher for heterosexual individuals who perceived e-cigarettes as less harmful than cigarettes (margin $=0.62$, $95 \% \mathrm{CI}=0.52,0.72)$ compared to sexual minority individuals (margin $=0.50,95 \% \mathrm{CI}=0.16,0.84$ ), who also perceived e-cigarettes as less harmful than cigarettes. The probability of e-cigarette use was higher among individuals who perceived e-cigarettes as less harmful for all heterosexual and sexual minority individuals. For heterosexual individuals, the highest probability of current e-cigarette use was observed among those who perceived e-cigarettes as less harmful (margin $=0.62,95 \% \mathrm{CI}=0.52,0.72)$ compared to those who were uncertain (margin $=0.27,95 \% \mathrm{CI}=0.13$, 0.41 ) or those who perceived them as just as harmful (margin $=0.20,95 \% \mathrm{CI}=0.11,0.28$ ), whereas the probability was not significant for individuals who perceived e-cigarettes as more harmful $($ margin $=0.06,95 \% \mathrm{CI}=-0.02,0.13)$. For the sexual minority individuals, current e-cigarette use probability was higher for those who perceived e-cigarettes as less harmful (margin $=0.50,95 \% \mathrm{CI}=0.16,0.84$ ) compared to just as harmful (margin $=0.32,95 \% \mathrm{CI}=0.05,0.59$ ), but the probabilities were not significant for more harmful (margin $=0.09,95 \% \mathrm{CI}=-0.12,0.30)$ or those who were uncertain $($ margin $=0.18,95 \% \mathrm{CI}=-0.06,0.43)$ in their e-cigarette perceptions. 
Table 1 Selected characteristics of a sample of US adults by current e-cigarette use (HINTS 2020 and 2019 data)

\begin{tabular}{|c|c|c|c|}
\hline & $\begin{array}{l}\text { Overall } \\
N(\%)\end{array}$ & $\begin{array}{l}\text { No current e-cigarette use } \\
n(\%)\end{array}$ & $\begin{array}{l}\text { Current daily or } \\
\text { some days e-cigarette } \\
\text { use } \\
n(\%)\end{array}$ \\
\hline Variable & $95,069,931(100 \%)$ & $65,837,038(69.25)$ & $29,232,893(30.75)$ \\
\hline \multicolumn{4}{|l|}{ Age } \\
\hline $18-25$ & $24,637,018(26.16)$ & $13,896,241(21.36)$ & $10,740,777(36.89)$ \\
\hline $26-34$ & $21,187,115(22.50)$ & $15,267,206(23.46)$ & $5,919,910(20.33)$ \\
\hline $35-49$ & $24,900,229(26.44)$ & $17,706,551(27.21)$ & $7,193,678(24.71)$ \\
\hline $50-64$ & $18,556,635(19.70)$ & $14,254,691(21.91)$ & $4,301,944(14.77)$ \\
\hline 65 or Older & $4,902,469(5.21)$ & $3,940,274(6.06)$ & $962,194(3.30)$ \\
\hline \multicolumn{4}{|l|}{ Sex } \\
\hline Female & $38,721,458(43.55)$ & $26,350,310(43.37)$ & $12,371,148(43.93)$ \\
\hline Male & $50,199,246(56.45)$ & $34,407,015(56.63)$ & $15,792,230(56.07)$ \\
\hline \multicolumn{4}{|l|}{ Race/ethnicity } \\
\hline Non-Hispanic White & $64,548,699(71.03)$ & $43,285,384(68.99)$ & $21,263,315(75.59)$ \\
\hline $\begin{array}{l}\text { Non-Hispanic Black/Afri- } \\
\text { can American }\end{array}$ & $5,616,931(6.18)$ & $3,936,114(6.27)$ & $1,680,817(5.98)$ \\
\hline Hispanic & $12,967,886(14.27)$ & $9,594,084(15.29)$ & $3,373,802(11.99)$ \\
\hline Non-Hispanic other & $7,742,559(8.52)$ & $5,929,818(9.45)$ & $1,812,741(6.44)$ \\
\hline \multicolumn{4}{|l|}{ Sexual identity } \\
\hline Heterosexual & $80,515,030(89.54)$ & $55,227,940(89.22)$ & $25,287,090(90.25)$ \\
\hline Sexual minority & $9,403,148(10.46)$ & $6,671,377(10.78)$ & $2,731,772(9.75)$ \\
\hline \multicolumn{4}{|l|}{ Level of education completed } \\
\hline Less than high school & $5,982,930(6.36)$ & $2,738,154(4.19)$ & $3,244,776(11.29)$ \\
\hline High school graduate & $22,645,949(24.08)$ & $16,127,429(24.70)$ & $6,518,520(22.68)$ \\
\hline Some college & $44,565,380(47.39)$ & $31,526,292(48.28)$ & $13,039,088(45.36)$ \\
\hline College graduate or higher & $20,852,232(22.17)$ & $14,909,454(22.83)$ & $5,942,777(20.67)$ \\
\hline \multicolumn{4}{|l|}{ Total family income } \\
\hline Less than $\$ 20,000$ & $17,675,640(19.77)$ & $12,972,036(21.17)$ & $4,703,604(16.71)$ \\
\hline$\$ 20,000$ to $<\$ 35,000$ & $8,294,432(9.28)$ & $4,996,400(8.15)$ & $3,298,033(11.72)$ \\
\hline$\$ 35,000$ to $<\$ 50,000$ & $13,421,714(15.01)$ & $8,773,551(14.32)$ & $4,648,163(16.52)$ \\
\hline$\$ 50,000$ to $<\$ 75,000$ & $18,129,521(20.27)$ & $12,475,895(20.36)$ & $5,653,626(20.09)$ \\
\hline$\$ 75,000$ or more & $31,902,775(35.68)$ & $22,060,840(36.00)$ & $9,841,935$ (34.97) \\
\hline \multicolumn{4}{|l|}{ Census region } \\
\hline Northeast & $14,559,871(15.32)$ & $10,405,955(15.81)$ & $4,153,916(14.21)$ \\
\hline Midwest & $21,510,132(22.63)$ & $14,708,004(22.34)$ & $6,802,128(23.27)$ \\
\hline West & $21,590,126(22.71)$ & $14,061,254(21.36)$ & $7,528,872(25.76)$ \\
\hline South & $37,409,802(39.35)$ & $26,661,826(40.50)$ & $10,747,976(36.77)$ \\
\hline \multicolumn{4}{|l|}{ General health status } \\
\hline Excellent/very good/good & $78,688,896(83.26)$ & $52,458,794(80.36)$ & $26,230,102(89.74)$ \\
\hline Fair or poor & $15,817,908(16.74)$ & $12,818,832(19.64)$ & 2,999,076 (10.26) \\
\hline \multicolumn{4}{|l|}{ Cigarette smoking status } \\
\hline Non-smoker & $31,024,280(32.92)$ & $22,649,234(34.80)$ & $8,375,046(28.73)$ \\
\hline Former smoker & $28,275,879(30.00)$ & $18,560,720(28.51)$ & $9,715,158(33.32)$ \\
\hline Current smoker & $34,947,278(37.08)$ & $23,883,257(36.69)$ & $11,064,022(37.95)$ \\
\hline \multicolumn{4}{|c|}{ Perceived harmfulness of e-cigarettes } \\
\hline Less harmful & $32,948,518(35.42)$ & $14,593,134(22.78)$ & $18,355,384(63.36)$ \\
\hline Just as harmful & $28,892,892(31.06)$ & $23,404,180(36.53)$ & $5,488,712(18.95)$ \\
\hline More harmful & $14,408,169(15.49)$ & $13,566,470(21.18)$ & $841,700(2.91)$ \\
\hline Uncertain & $16,785,358(18.04)$ & $12,503,206(19.52)$ & $4,282,151(14.78)$ \\
\hline
\end{tabular}

Data source: 2020 and 2019 Health Information National Trends Survey (HINTS 5, Cycles 4 and 3) Weighted $N=95,069,931$ 
Table 2 Adjusted odds ratios of e-cigarette use status associated with sociodemographic characteristics, cigarette smoking status, and perceived harmfulness of e-cigarettes (Weighted $N=95,069,931$ )

\begin{tabular}{|c|c|c|}
\hline Variable & AOR & $95 \% \mathrm{CI}$ \\
\hline \multicolumn{3}{|l|}{ Age } \\
\hline $18-25$ & Ref & - \\
\hline $26-34$ & $0.32 *$ & $(0.11,0.88)$ \\
\hline $35-49$ & 0.37 & $(0.12,1.17)$ \\
\hline $50-64$ & $0.23 * *$ & $(0.07,0.77)$ \\
\hline 65 or older & $0.17 *$ & $(0.03,0.80)$ \\
\hline \multicolumn{3}{|l|}{ Sex } \\
\hline Female & Ref & - \\
\hline Male & 0.62 & $(0.32,1.21)$ \\
\hline \multicolumn{3}{|l|}{ Race/ethnicity } \\
\hline Non-Hispanic White & Ref & - \\
\hline Non-Hispanic Black/African American & 1.76 & $(0.49,6.39)$ \\
\hline Hispanic & 1.15 & $(0.48,2.73)$ \\
\hline Non-Hispanic others & 1.20 & $(0.41,3.47)$ \\
\hline \multicolumn{3}{|l|}{ Sexual identity } \\
\hline Heterosexual (i.e., straight) & Ref & - \\
\hline $\begin{array}{l}\text { Sexual minorities (Lesbian/gay or } \\
\text { bisexual) }\end{array}$ & 0.95 & $(0.34,2.65)$ \\
\hline \multicolumn{3}{|l|}{ Level of education completed } \\
\hline Less than High School & Ref & - \\
\hline High School graduate & 0.36 & $(0.08,1.70)$ \\
\hline Some college & 0.41 & $(0.10,1.67)$ \\
\hline College graduate or higher & 0.25 & $(0.05,1.17)$ \\
\hline \multicolumn{3}{|l|}{ Total family income } \\
\hline Less than $\$ 20,000$ & Ref & - \\
\hline$\$ 20,000$ to $<\$ 35,000$ & 2.54 & $(0.79,8.18)$ \\
\hline$\$ 35,000$ to $<\$ 50,000$ & 2.51 & $(0.83,7.63)$ \\
\hline$\$ 50,000$ to $<\$ 75,000$ & 1.40 & $(0.56,3.51)$ \\
\hline$\$ 75,000$ or more & 1.44 & $(0.56,3.73)$ \\
\hline \multicolumn{3}{|l|}{ Census region } \\
\hline Northeast & Ref & - \\
\hline Midwest & 1.25 & $(0.43,3.61)$ \\
\hline West & 1.69 & $(0.66,4.35)$ \\
\hline South & 1.27 & $(0.51,3.16)$ \\
\hline \multicolumn{3}{|l|}{ General health status } \\
\hline Excellent/very good/good & Ref & - \\
\hline Fair or poor & $0.32 * *$ & $(0.15,0.69)$ \\
\hline \multicolumn{3}{|l|}{ Cigarette smoking status } \\
\hline Non-smoker & Ref & - \\
\hline Former smoker & 2.50 & $(0.97,6.47)$ \\
\hline Current smoker & $3.48 * *$ & $(1.25,9.72)$ \\
\hline \multicolumn{3}{|l|}{ Perceived harmfulness of e-cigarettes } \\
\hline Less harmful & Ref & - \\
\hline Just as harmful & $0.13 * * *$ & $(0.06,0.27)$ \\
\hline More harmful & $0.03 * * *$ & $(0.01,0.10)$ \\
\hline Uncertain & $0.18 * * *$ & $(0.08,0.45)$ \\
\hline
\end{tabular}

AOR, adjusted odds ratio; 95\% CI, 95\% confidence interval; Ref, reference group

${ }^{*} p<0.05, * * p<0.01, * * * p<0.001$

\section{Discussion}

This study used recent nationally representative sample data to assess whether perceived harmfulness of e-cigarettes is associated with e-cigarette use, and if so, whether this association is conditioned on cigarette smoking status or sexual identity. Compared to earlier studies that reported a prevalence of $0.3 \%$ in 2010 and $4.5 \%$ in 2019 of current e-cigarette use (Cornelius et al. 2020; McMillen et al. 2014), we found a higher prevalence of current use of e-cigarettes daily or some days (30.75\%) among the U.S. population. This implies that the prevalence of e-cigarette use has increased approximately 6.8 -fold during 2020 , which was during the coronavirus disease 2019 (COVID-19) pandemic and related lockdown orders. A possible explanation is that the COVID19 stressors, such as increased anxiety, social isolation, and economic concerns, may have increased tobacco use, including current e-cigarette use (Smith et al. 2020; Van Zyl-Smit et al. 2020; Yach 2020). Similarly, our findings showed that the number of individuals who perceived e-cigarettes to be less harmful has increased $(35.42 \%)$ relative to the previous prevalence of $34.5 \%$ in 2017 (Huang et al. 2019). This perception has almost doubled (63.36\%) among current e-cigarette users. Current e-cigarette use also has increased among current cigarette smokers, at $37.95 \%$, compared to $36.9 \%$ in 2019 (Cornelius et al. 2020). The increase also was observed among sexual minorities, for whom approximately 1 in 10 self-identified as lesbian, gay, or bisexual, compared to $7 \%$ in 2018, who reported e-cigarette use (Rapaport 2018). The findings revealed that approximately 1 in 10 of the population who rate their general health status as fair or poor are likely to be current e-cigarette users. The results suggest the need to consider perceived health risks and behavioral or lifestyle modifications for public health interventions as well as research on e-cigarette use.

The results from our multivariable analysis showed significant differences in e-cigarette use between age groups. In alignment with other studies (Ganz et al. 2018; Johnson et al. 2016), we found that individuals within the age range of 18-25 years were more likely to engage in e-cigarette use daily or some days than were individuals within the age ranges 26-34 and 50+ years. We did not, however, find significant differences in e-cigarette use between the age groups 18-25 and 35-49 years. This finding suggests that, although the prevalence of e-cigarette use may be higher among individuals aged 18-25 years, the difference in the risks of engaging in e-cigarette use may not be significant between them and those aged 35-49 years. In contrast with a previous study that used a nationally representative sample of adults (Emory et al. 2016), e-cigarette use did not differ significantly between sexual minority and heterosexual groups in our study. The differences in the findings from 
Fig. 1 Differences in e-cigarette use between and within cigarette smoking status and perceived harmfulness of e-cigarettes
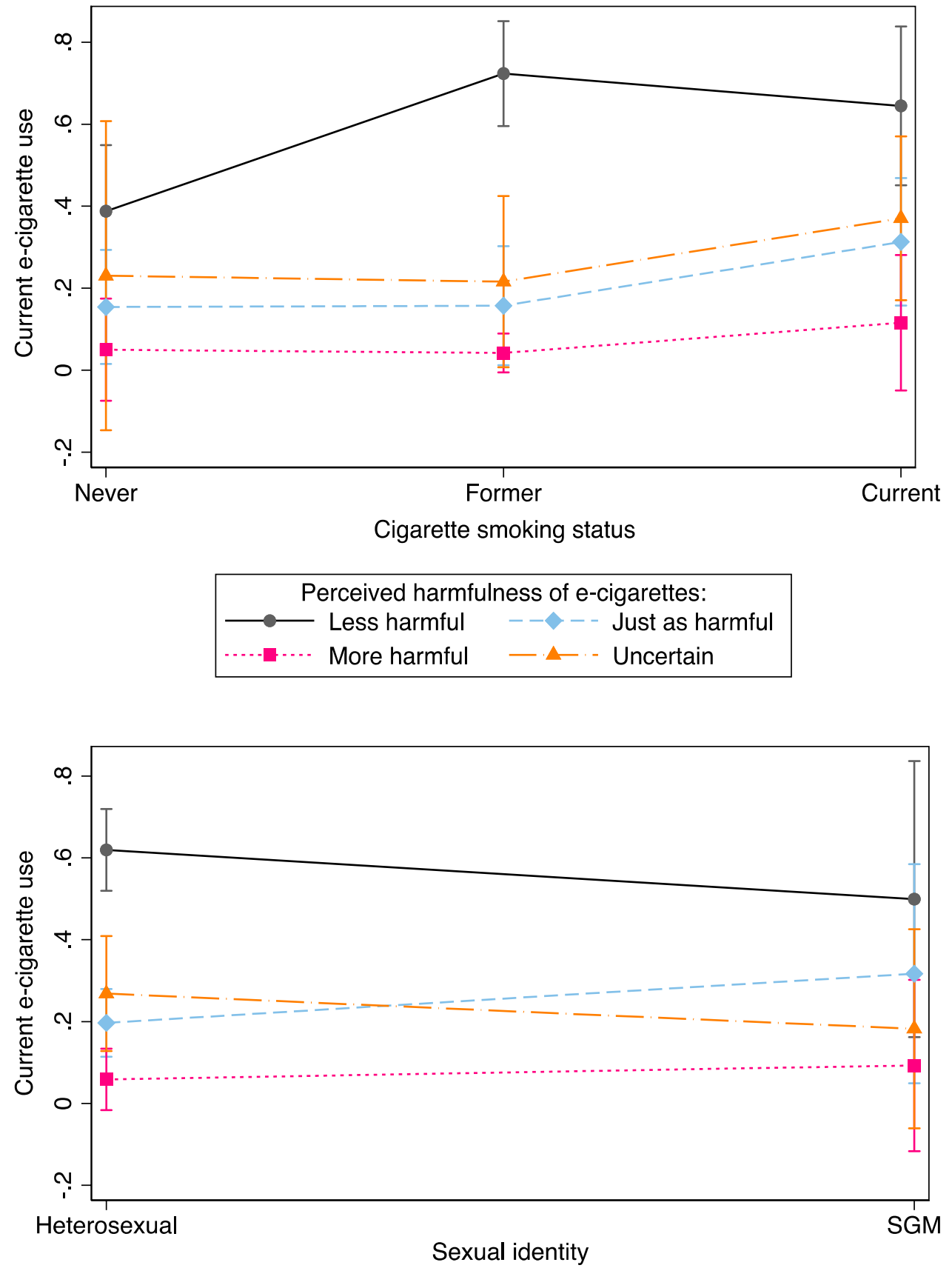

Fig. 2 Differences in e-cigarette use between and within sexual identity and perceived harmfulness of e-cigarettes these studies may be due to differences in survey administration characteristics and participants. For instance, the data analyzed by Emory et al. (2016) for their study was collected in 2013 and included only $4.8 \%$ sexual minorities, whereas our data were collected in 2019 and 2020, and $10.46 \%$ of the sample was self-identified as lesbian, gay, or bisexual. These differences may have accounted for the differences in the findings between the studies. It also may be that, regardless of SGM status, e-cigarette use may be normalized by certain social contexts, including more prevalent peer e-cigarette use (Blosnich et al. 2013; Jannat-Khah et al. 2018), environments of advertising e-cigarettes or tobacco companies' strategies (Dilley et al. 2008; Fallin et al. 2015; U.S. National Cancer Institute 2017), or marketing efforts 
of e-cigarettes as products that can be used as a means to circumvent smoke-free laws (Grana and Ling 2014), all of which may not influence the difference in e-cigarette use by SGM status. Our findings revealed, however, that the association between current e-cigarette use and perceived harmfulness of e-cigarettes was influenced by sexual identity. Contrary to previous studies (Ganz et al. 2018; Nayak et al. 2017), we found that, for sexual minority individuals or heterosexual individuals, those who perceived e-cigarettes as less harmful were more likely to engage in current e-cigarette use.

General health status may have implications for engaging in daily or some days e-cigarette use. Our findings revealed that individuals who had fair or poor general health status were less likely to use e-cigarettes daily or some days compared to those who had excellent, very good, or good general health status. This association implies that individuals who have fair or poor health conditions may be concerned about their health and, therefore, may quit or avoid e-cigarette use to protect their health. Consistently, Li et al. (2019) found that individuals with poor health conditions were less likely to engage in smoking and more likely to report quit attempts due to cancer, heart disease, or chronic lung conditions.

Our findings further revealed that lower perceived harmfulness of e-cigarettes was associated with e-cigarette use daily or some days. These findings are consistent with previous studies (Amrock et al. 2016; Huang et al. 2019; Manzione et al. 2020) that found that a reason for continuous use of e-cigarettes was related mainly to less perceived harmfulness of e-cigarettes. We also found that current cigarette smokers were more likely to engage in e-cigarettes use than were non-cigarette smokers. A possible explanation for these findings is that current cigarette smokers may be using e-cigarettes with an intention or attempt to quit cigarette smoking. Thus, these individuals may be engaging in dual-use of e-cigarettes and conventional cigarettes to either compensate for their cravings or perception of e-cigarettes as less harmful than cigarettes (Miller et al. 2020; Rhoades et al. 2019). This dual-use may increase the health risks associated with nicotine products among the population (CDC 2020; Singh et al. 2020; U.S. Department of Health and Human Services 2016).

Our study showed that the association between current e-cigarette use and perceived harmfulness of e-cigarettes varied by cigarette smoking status among U.S. adults, suggesting differences in e-cigarette use between and within cigarette smoking status and perceived harmfulness of e-cigarettes. Consistent with previous studies (Ganz et al. 2018; Popova et al. 2018), we observed that former and current cigarette smokers who perceived e-cigarettes as less harmful than cigarettes had a higher probability of using e-cigarettes daily or some days than did non-cigarette smokers. Perhaps e-cigarette use among cigarette smokers is due to a positive affect toward tobacco use, including e-cigarettes, which, in turn, increases the lower perception of harm from e-cigarettes during the COVID-19 pandemic, as individuals may be using tobacco products as stress and anxiety coping mechanisms during the COVID-19 pandemic (Popova et al. 2018; Yach 2020). The COVID-19 stressors and coping mechanisms may further explain our findings that, although current and former cigarette smokers who perceived e-cigarettes as less harmful were more likely to engage in current e-cigarette use behavior than those who were uncertain or perceived e-cigarettes as just as harmful, there were no significant differences for current and former smokers who perceived e-cigarettes as more harmful. Similarly, non-cigarette smokers who perceived e-cigarettes as less harmful had a higher likelihood of current e-cigarette use than did those who perceived it as just as harmful. The likelihoods were, however, not significant for those who perceived e-cigarettes as more harmful or were uncertain. Overall, these findings imply a negative association between cigarette smoking and lower perception of e-cigarettes for current daily or some days e-cigarette use behavior. A plausible increase in harm perception of e-cigarettes and cigarettes among cigarette smokers may be associated with a lower likelihood of e-cigarette use, including a switch from cigarettes to e-cigarettes among smokers and vice versa (Huang et al. 2019). Therefore, public health professionals should consider the risks of cigarette smoking in addressing the lower perceived harmfulness of e-cigarettes among daily or some days e-cigarette users.

This study has some limitations that need to be considered. This is a cross-sectional study and provides only a snapshot of the prevalence of e-cigarette use and its risks factors, without consideration of the temporal trends and associations. In addition, the study used self-reported data and, thus, may include recall and participants' biases. These biases may potentially result in over- or underreporting, which could have affected our findings. Nevertheless, the HINTS questions used to assess the self-reported responses have been widely adopted since 2003. Moreover, e-cigarette use and its risk perceptions are rapidly evolving; therefore, cross-sectional study design and snapshot results may be necessary to understand e-cigarette use dynamics, which may have policy and intervention implications for the continued use of e-cigarettes. In addition, this study included only noninstitutionalized adults, which does not allow for generalizing the study findings to youth and institutionalized adults. Nonetheless, this study included a recent nationally representative sample, which makes the findings important and generalizable to the general U.S. adult population. 


\section{Conclusion}

Our study demonstrated that daily or some days use of e-cigarettes is associated with current cigarette smoking and less perceived harmfulness of e-cigarettes among the U.S. adult population. Young adults were more likely to engage in current e-cigarette use behavior. The study findings did not, however, support the notion that current e-cigarette use differs significantly between sexual minority and heterosexual groups. Lower perceptions of the harm of e-cigarettes relative to cigarettes among cigarette smokers and sexual identity groups was associated with a higher level of engagement in current e-cigarette use behavior. This positive association between current cigarette smoking and daily or some days use of e-cigarettes in the population implies that individuals may have been plausibly engaging in the dual-use of e-cigarettes and conventional cigarettes, possibly either to compensate for their cravings or due to their perception of e-cigarettes as less harmful than cigarettes. These findings highlight the need to enhance tailored health promotion and intervention efforts to reduce the potential perceived health risks and behavioral or lifestyle risks related to the use of tobacco products, especially e-cigarette use daily or some days among young adults and individuals with excellent or good general health status. Specifically, increasing harm perceptions of e-cigarettes and cigarettes among cigarette smokers and sexual identity subgroups (lesbian, gay, bisexual, and heterosexual) may reduce or prevent initiation or continuous use of e-cigarettes and other tobacco products (Huang et al. 2019). Finally, research is needed to identify different new designs and types of e-cigarettes, as well as dual-use, to better understand e-cigarette use dynamics in the population.

Author contributions David Adzrago served as the leading author, conceived the study, performed the statistical analyses, conducted the literature review, drafted the manuscript, and coordinated writing the manuscript. Yue Shi and Kayo Fujimoto contributed to the writing of the manuscript and provided critical revisions to the manuscript. All authors reviewed the manuscript and agreed to the publication of the manuscript.

Availability of data and material Publicly available data and material at https://hints.cancer.gov.

Code availability Not applicable.

\section{Declarations}

Conflicts of interest All authors have no conflicts of interest to disclosure that are relevant to the content of this article.
Ethics approval Because we used the HINTS de-identified publicly available data, the authors' Institutional Review Board determined that this study is not a research with human subjects and therefore qualified for an exemption (RE: HSC-SPH-21-0784).

Consent to participate Not applicable.

\section{References}

American Psychological Association (2015) Guidelines for psychological practice with transgender and gender nonconforming people. Am Psychol 70(9):832-864

Amrock SM, Lee L, Weitzman M (2016) Perceptions of e-cigarettes and noncigarette tobacco products among US youth. Pediatrics 138(5):e20154306. https://doi.org/10.1542/peds.2015-4306

Blosnich J, Lee JG, Horn K (2013) A systematic review of the aetiology of tobacco disparities for sexual minorities. Tob Control 22(2):66-73

Buchting FO, Emory KT, Kim Y, Fagan P, Vera LE, Emery S (2017) Transgender use of cigarettes, cigars, and e-cigarettes in a national study. Am J Prev Med 53(1):e1-e7

Centers for Disease Control and Prevention (2020) Quick facts on the risks of e-cigarettes for kids, teens, and young adults. Smoking \& Tobacco Use. https://www.cdc.gov/tobacco/basic_information/ e-cigarettes/Quick-Facts-on-the-Risks-of-E-cigarettes-for-KidsTeens-and-Young-Adults.html. Accessed 16 Jan 2020

Cobb NK, Brookover J, Cobb CO (2015) Forensic analysis of online marketing for electronic nicotine delivery systems. Tob Control 24(2):128-131

Cooper M, Case KR, Loukas A, Creamer MR, Perry CL (2016) E-cigarette dual users, exclusive users and perceptions of tobacco products. Am J Health Behav 40(1):108-116. https://doi.org/10.5993/ AJHB.40.1.12

Cornelius ME, Wang TW, Jamal A, Loretan CG, Neff LJ (2020) Tobacco product use among adults-United States, 2019. Morb Mortal Wkly Rep 69(46):1736

Czoli CD, Fong GT, Mays D, Hammond D (2017) How do consumers perceive differences in risk across nicotine products? A review of relative risk perceptions across smokeless tobacco, e-cigarettes, nicotine replacement therapy and combustible cigarettes. Tob Control 26(e1):e49-e58

Delahanty J, Ganz O, Hoffman L, Guillory J, Crankshaw E, Farrelly M (2019) Tobacco use among lesbian, gay, bisexual and transgender young adults varies by sexual and gender identity. Drug Alcohol Depend 201:161-170

Dilley JA, Spigner C, Boysun MJ, Dent CW, Pizacani BA (2008) Does tobacco industry marketing excessively impact lesbian, gay and bisexual communities? Tob Control 17(6):385-390

Douglass B, Solecki S, Fay-Hillier T (2020) The harmful consequences of vaping: a public health threat. J Addict Nurs 31(2):79-84

Emory K, Kim Y, Buchting F, Vera L, Huang J, Emery SL (2016) Intragroup variance in lesbian, gay, and bisexual tobacco use behaviors: evidence that subgroups matter, notably bisexual women. Nicotine Tob Res 18(6):1494-1501. https://doi.org/10.1093/ntr/ntv208

Fallin A, Goodin A, Lee YO, Bennett K (2015) Smoking characteristics among lesbian, gay, and bisexual adults. Prev Med 74:123-130

Finney Rutten LJ, Blake KD, Skolnick VG, Davis T, Moser RP, Hesse BW (2020) Data resource profile: the national cancer institute's health information national trends survey (HINTS). Int J Epidemiol 49(1):17-17j 
Ganz O, Johnson AL, Cohn AM, Rath J, Horn K, Vallone D, Villanti AC (2018) Tobacco harm perceptions and use among sexual and gender minorities: findings from a national sample of young adults in the United States. Addict Behav 81:104-108. https:// doi.org/10.1016/j.addbeh.2018.01.032

Grana RA, Ling PM (2014) "Smoking revolution": a content analysis of electronic cigarette retail websites. Am J Prev Med 46(4):395-403

Grana R, Benowitz N, Glantz SA (2014) E-cigarettes: a scientific review. Circ 129(19):1972-1986. https://doi.org/10.1161/CIRCU LATIONAHA.114.007667

Huang J, Feng B, Weaver SR, Pechacek TF, Slovic P, Eriksen MP (2019) Changing perceptions of harm of e-cigarette vs cigarette use among adults in 2 US National Surveys from 2012 to 2017. JAMA Netw Open 2(3):e191047. https://doi.org/10.1001/jaman etworkopen.2019.1047

Jamal A, Phillips E, Gentzke AS, Homa DM, Babb SD, King BA, Neff LJ (2018) Current cigarette smoking among adults-United States, 2016. Morb Mortal Wkly Rep 67(2):53

Jannat-Khah DP, Dill L.J, Reynolds SA, Joseph MA (2018) Stress, socializing, and other motivations for smoking among the lesbian, gay, bisexual, transgender, and queer community in New York City. Am J Health Promot 32(5):1178-1186

Johnson SE, Holder-Hayes E, Tessman GK, King BA, Alexander T, Zhao X (2016) Tobacco product use among sexual minority adults: Findings from the 2012-2013 national adult tobacco survey. Am J Prev Med 50(4):e91-e100

Li L, Borland R, O'Connor RJ, Fong GT, McNeill A, Driezen P, Cummings MK (2019) The association between smokers' self-reported health problems and quitting: findings from the ITC four country smoking and vaping wave 1 survey. Tob Prev Cessation 5:49

Luo C, Zheng X, Zeng DD, Leischow S (2014) Portrayal of electronic cigarettes on YouTube. BMC Public Health 14(1):1028

Manzione LC, Shan L, Azagba S (2020) Associations between risk perceptions and cigarette, e-cigarette, and dual-product use among Canadian adolescents. Tob Use Insights 13:1179173X20903784. https://doi.org/10.1177/1179173X20903784

McMillen RC, Gottlieb MA, Shaefer RMW, Winickoff JP, Klein JD (2014) Trends in electronic cigarette use among U.S. adults: use is increasing in both smokers and nonsmokers. Nicotine Tob Res 17(10): 1195-1202

Miller CR, Smith DM, Goniewicz ML (2020) Changes in nicotine product use among dual users of tobacco and electronic cigarettes: findings from the population assessment of tobacco and health (PATH) study, 2013-2015. Subst Use Misuse 55(6):909-913

National Academies of Sciences, Engineering, and Medicine (2018) Public health consequences of e-cigarettes. Washington, DC: National Academies Press. https://books.google.com/books?hl= en $\& \mathrm{l}=\& \mathrm{id}=\mathrm{jQhgDwAAQBAJ} \& \mathrm{oi}=$ fnd $\& \mathrm{pg}=\mathrm{PR} 1 \& \mathrm{dq}=$ Public + Health+Consequences + of + e-Cigarettes. $+\&$ ots $=$ owbATK-92E \& sig=-syDuF3SXMcKhsheVoViKyRpDUM\#v=onepage $\& \mathrm{q}=$ Public $\% 20$ Health $\% 20$ Consequences $\% 20$ of $\% 20$-Cigarettes.\&f $=$ false. Accessed 17 May 2021

Nayak P, Salazar LF, Kota KK, Pechacek TF (2017) Prevalence of use and perceptions of risk of novel and other alternative tobacco products among sexual minority adults: results from an online national survey, 2014-2015. Prev Med 104:71-78. https://doi.org/ 10.1016/j.ypmed.2017.05.024

Noel JK, Rees VW, Connolly GN (2011) Electronic cigarettes: a new 'tobacco'industry? Tob Control 20(1):81-81

Ortiz K, Mamkherzi J, Salloum R, Matthews AK, Maziak W (2017) Waterpipe tobacco smoking among sexual minorities in the United States: evidence from the National Adult Tobacco Survey (2012-2014). Addict Behav 74:98-105

Paek HJ, Kim S, Hove T, Huh JY (2014) Reduced harm or another gateway to smoking? Source, message, and information characteristics of e-cigarette videos on YouTube. J Health Commun 19(5):545-560

Pericot-Valverde I, Gaalema DE, Priest JS, Higgins ST (2017) E-cigarette awareness, perceived harmfulness, and ever use among US adults. Prev Med 104:92-99

Perski O, Beard E, Brown J (2020) Association between changes in harm perceptions and e-cigarette use among current tobacco smokers in England: a time series analysis. BMC Med 18:1-10

Piñeiro B, Correa JB, Simmons VN, Harrell PT, Menzie NS, Unrod M, Meltzer LR, Brandon TH (2016) Gender differences in use and expectancies of e-cigarettes: online survey results. Addict Behav 52:91-97. https://doi.org/10.1016/j.addbeh.2015.09.006

Pisinger C, Døssing M (2014) A systematic review of health effects of electronic cigarettes. Prev Med 69:248-260

Popova L, Owusu D, Weaver SR, Kemp CB, Mertz CK, Pechacek TF, Slovic P (2018) Affect, risk perception, and the use of cigarettes and e-cigarettes: a population study of U.S. adults. BMC Public Health 18(1):395. https://doi.org/10.1186/ s12889-018-5306-Z

Popova L, So J, Sangalang A, Neilands TB, Ling PM (2017) Do emotions spark interest in alternative tobacco products? Health Educ Behav 44(4):598-612. https://doi.org/10.1177/10901 98116683169

Rapaport L (2018) Almost one in 20 U.S. adults now use e-cigarettes. Reuters health. https://www.reuters.com/article/us-health-ecigsus-adults/almost-one-in-20-u-s-adults-now-use-e-cigarettesidUSKCN1LC2DN. Accessed 27 Aug 2021

Rhoades DA, Comiford AL, Dvorak JD, Ding K, Hopkins M, Spicer P, Wagener TL, Doescher MP (2019) Vaping patterns, nicotine dependence and reasons for vaping among American Indian dual users of cigarettes and electronic cigarettes. BMC Public Health 19(1):1-8

Singh S, Windle SB, Filion KB, Thombs BD, O'Loughlin JL, Grad R, Eisenberg MJ (2020) E-cigarettes and youth: patterns of use, potential harms, and recommendations. Prev Med 133:106009

Smith L, Jacob L, Yakkundi A, McDermott D, Armstrong NC, Barnett Y, Lopez-Sanchez GF, Martin S, Butler L, Tully MA (2020) Correlates of symptoms of anxiety and depression and mental wellbeing associated with COVID-19: a cross-sectional study of UK-based respondents. Psychiatry Res 291:113138

StataCorp LLC (2019) Stata statistical software: release 16. StataCorp LLC, College Station

Tan AS, Bigman CA (2014) E-cigarette awareness and perceived harmfulness: prevalence and associations with smoking-cessation outcomes. Am J Prev Med 47(2):141-149

U.S. Department of Health and Human Services (2016) E-cigarette use among youth and young adults: a report of the surgeon general. Atlanta: U.S. Department of Health and Human Services, CDC https://www.cdc.gov/tobacco/data_statistics/sgr/e-cigar ettes/pdfs/2016_sgr_entire_report_508.pdf. Accessed 8 Jan 2020

U.S. Department of Health and Human Services (2014) The health consequences of smoking - 50 years of progress: a report of the surgeon general. Atlanta, GA: U.S. Department of Health and Human Services, CDC. https://aahb.org/Resources/Pictu res/Meetings/2014-Charleston/PPT\%20Presentations/Sunday\%20Welcome/Abrams.AAHB.3.13.v1.o.pdf. Accessed 17 May 2021

U.S. National Cancer Institute (2017) A socioecological approach to addressing tobacco-related health disparities. National Cancer Institute Tobacco Control Monograph 22

Van Zyl-Smit RN, Richards G, Leone FT (2020) Tobacco smoking and COVID-19 infection. Lancet Respir Med 8(7):664-665 
Villarroel MA, Cha AE, Vahratian A (2020) Electronic cigarette use among US adults, 2018. https://stacks.cdc.gov/view/cdc/87918. Accessed 17 May 2021

Wackowski OA, Delnevo CD (2016) Young adults' risk perceptions of various tobacco products relative to cigarettes: results from the National Young Adult Health Survey. Health Educ Behav 43(3):328-336. https://doi.org/10.1177/1090198115599988

Westat (2020) Health information National Trends Survey 5 (HINTS 5): cycle 4 methodology report. Rockville, MD: Westat. https:// hints.cancer.gov/docs/methodologyreports/HINTS5_Cycle4_ MethodologyReport.pdf. Accessed 1 Apr 2021
Wheldon CW, Wiseman KP (2019) Tobacco use among transgender and gender non-conforming adults in the United States. Tob Use Insights 12:1179173X19849419

Yach D (2020) Tobacco use patterns in five countries during the COVID-19 lockdown. Nicotine Tob Res 22(9):1671-1672

Publisher's note Springer Nature remains neutral with regard to jurisdictional claims in published maps and institutional affiliations. 